\title{
QUESTÕES SOCIOECONÔMICO-FAMILIARES ASSOCIADAS À PRÁTICA SEXUAL DE ADOLESCENTES: UM ESTUDO DA CAPITAL DE MATO GROSSO*
}

\author{
Débora de Medeiros Bagli1', Shayera Miranda², Christine Baccarat Godoy Martins², Karla Fonseca de Matos ${ }^{3}$
}

\begin{abstract}
RESUMO: A adolescência é uma fase da vida humana caracterizada por grandes mudanças físicas, biológicas e comportamentais. O presente estudo objetivou estudar as questões socioeconômico-culturais relativas à prática sexual de adolescentes, através de pesquisa de caráter quantitativo, realizada no primeiro semestre de 2010, em cinco escolas estaduais do Município de Cuiabá - Mato Grosso. A população estudada foi composta por 499 alunos do primeiro ano do ensino médio. Os resultados mostraram que não houve diferença significativa entre a prática sexual dos adolescentes pesquisados e suas condições socioeconômico-familiares, com exceção àquelas relacionadas com a situação conjugal dos pais; com o fato do adolescente trabalhar, ou não, bem como se faz uso de contraceptivo. Os resultados apontam para a necessidade de gestores, educadores e profissionais trabalhar a sexualidade com os adolescentes nessa fase de muitas transformações e dúvidas. PALAVRAS-CHAVE: Sexualidade; Saúde do adolescente; Adolescente; Comportamento do adolescente; Comportamento sexual.
\end{abstract}

\section{FAMILY-SOCIO-ECONOMIC QUESTIONS ASSOCIATED WITH ADOLESCENTS' SEXUAL PRACTICES: A STUDY FROM THE CAPITAL OF MATO GROSSO}

\begin{abstract}
Adolescence is a phase of human life characterized by great physical, biological and behavioral changes. This study aimed to study socio-economic and cultural questions relative to adolescents' sexual practices: this was achieved via quantitative research carried out in the first semester of 2010, in five state schools in the county of Cuiabá in Mato Grosso. The sample-population was composed of 499 students from the first year of middle school. The results showed that there was no significant difference between the sexual practices of the adolescents researched and their socio-economic or family conditions, with the exception of those related to the conjugal situation of parents; with the fact of the adolescent working or not; and whether he or she used contraceptives. The results indicate the need for managers, educators and healthcare professionals to collaborate with adolescents on sexual health issues in this phase of so many changes and doubts.

KEYWORDS: Sexuality; Adolescent health; Adolescent; Adolescent behavior; Sexual behavior.

\section{CUESTIONES SOCIOECONÓMICAS Y FAMILIARES ASOCIADAS A LA PRÁCTICA SEXUAL DE ADOLESCENTES: UN ESTUDIO DE LA CAPITAL DE MATO GROSSO}

\begin{abstract}
RESUMEN: La adolescencia es una fase de la vida humana caracterizada por grandes cambios físicos, biológicos y comportamentales. Este estudio tuvo el objetivo de estudiar las cuestiones socioeconómico-culturales acerca de la práctica sexual de adolescentes, por medio de investigación de carácter cuantitativo, realizada en el primer semestre de 2010, en cinco escuelas estaduales de municipio de Cuiabá - Mato Grosso. La población estudiada se compuso de 499 alumnos del primero año del nivel medio. Los resultados mostraron que no hubo diferencia significativa entre la práctica sexual de los adolescentes de la investigación y sus condiciones socioeconómico-familiares, con excepción de aquellas relacionadas con la situación conjugal de los padres; con el hecho del adolescente trabajar o no, así como se él hace uso del contraceptivo. Los resultados apuntan para la necesidad de gestores, educadores y profesionales de trabajar la sexualidad con los adolescentes en esa fase de muchas transformaciones y dudas. PALABRAS-CLAVE: Sexualidad; Salud del adolescente; Adolescente; Comportamiento del adolescente; Comportamiento sexual.
\end{abstract}

*Pesquisa financiada pela Fundação de Amparo à Pesquisa de Mato Grosso - FAPEMAT.

${ }^{1}$ Acadêmica de Enfermagem da Universidade Federal de Mato Grosso-UFMT.

${ }^{2}$ Enfermeira. Doutora em Saúde Pública. Professora do Departamento de Enfermagem da UFMT.

${ }^{3}$ Enfermeira. Mestre em Enfermagem.

Autor correspondente:

Christine Baccarat Godoy Martins

Universidade Federal do Mato Grosso

Rua Fortaleza, 70 - CEP - 78065-350 - Cuiabá-MT-Brasil

E-mail: leocris2001@terra.com.br
Recebido: 08/01/2011

Aprovado: 31/08/2011 


\section{INTRODUÇÃO}

A adolescência é uma fase da vida humana caracterizada por grandes mudanças físicas, biológicas e comportamentais; período de descobertas e de transição para a maturidade, marcada pela busca da identidade, pelo questionamento frente às dúvidas, pela identificação por grupos e pela evolução da sexualidade ${ }^{(1)}$.

Neste processo de construção da identidade do adolescente, a sexualidade se apresenta como elemento significativo, manifestada pela identificação com a imagem corporal, pela descoberta do outro como objeto de amor ou desejo, pelas relações com os familiares e com os grupos de convívio ${ }^{(2)}$. Somando-se a estas questões, estudo aponta as questões socioeconômicas e familiares como fatores que interferem na forma de como o jovem vivencia sua sexualidade ${ }^{(3)}$.

Entre as condições socioeconômico e familiares com reflexo na sexualidade do adolescente, encontram-se o nível de escolaridade dos pais, as condições de vida, como o desemprego e habitação, densidade familiar, acesso aos serviços de saúde e renda. Estes fatores resultam em maior ou menor exposição à doenças físicas, estresse familiar e suporte social insuficiente para o exercício da sexualidade sem $\operatorname{riscos}^{(4)}$.

A importância da família ou grupo social em que o adolescente está inserido, juntamente com as condições socioeconômicas, contribuem significativamente para a sua formação, uma vez que será através desse conjunto de fatores que o jovem encontrará o apoio e a liberdade de discussão sobre a sexualidade. O não-acesso a essas informações e a falta de vínculo familiar associada à condições econômicas precárias desencadeiam na descoberta precoce da sexualidade, possível exposição às doenças sexualmente transmissíveis e gravidez não planejada ${ }^{(5)}$. Desse modo, o presente objetivou estudar as questões socioeconômico-familiares relativas à prática sexual de adolescentes.

\section{METODOLOGIA}

A hipótese do estudo foi de que existe associação entre as questões socioeconômico-familiares e o exercício da sexualidade de adolescentes. Trata-se de um estudo quantitativo e transversal realizado em cinco escolas estaduais do Município de Cuiabá, Estado do Mato Grosso, no primeiro semestre de 2010, cuja população estudada foi composta por 499 alunos do primeiro ano do ensino médio. O critério de inclusão foi estar na faixa etária de 11 a 19 anos, período considerado pela
Organização Mundial da Saúde como da adolescência. As escolas, bem como a Secretaria de Estado de Educação, autorizaram por escrito a realização do estudo. A autorização dos pais ou responsável foi providenciada antecipadamente pela direção das escolas envolvidas, não havendo nenhuma recusa. Os adolescentes foram convidados a participar da pesquisa assinando o Termo de Consentimento Livre e Esclarecido.

Para a coleta de dados foi aplicado um questionário estruturado, composto por 19 questões fechadas, depositados diretamente em urnas distribuídas pelas salas de aulas, a fim de garantir o anonimato. Os dados foram armazenados e analisados pelo programa Epi Info versão 3.5.1, com análises bivariadas, em que se considerou valor de $p<0,05$ para associação estatisticamente significativa.

Foram estudadas as seguintes variáveis de exposição: a escolaridade da mãe, a renda familiar, a situação conjugal dos pais com quem o adolescente residia, se o adolescente trabalhava, se era praticante de alguma religião, e se este adolescente considerava-se amado pelos pais. Foram consideradas como variáveis de desfecho: a iniciação da vida sexual, a vida sexual ativa ou não, a frequência na prática do sexo, a experiência da gravidez, a presença de doença sexualmente transmissível, o uso de método de contracepção e a vitimização de violência sexual.

Para a análise bivariada, em que se buscaram associações entre variáveis, foi utilizado o teste de Qui-quadrado, em que se considerou a probabilidade das associações se dar ao acaso menor que $5 \%$ (valor de $\mathrm{p}<0,05$ ), com um intervalo de confiança de $95 \%$, o que aponta para associações estatisticamente significativas.

O presente estudo está inserido em uma pesquisa matricial aprovada pelo Comitê de Ética do Hospital Universitário Júlio Muller, sob o protocolo número 613 /CEP - HUJM/09. Do questionário da pesquisa foram selecionadas, para este artigo, as questões relativas ao contexto socioeconômico-familiar.

\section{RESULTADOS}

Foram estudados $190(38,1 \%)$ meninos e 309 (61,9\%) meninas. Observou-se que, entre os adolescentes cujos pais são casados, a maioria $(60,3 \%)$ ainda não teve relação sexual (Tabela 1). Por outro lado, entre aqueles em que a mãe é ausente (sem contato com o filho) ou os pais são namorados (não moram juntos), quase a totalidade já teve sua iniciação sexual (100\% e $80 \%$, respectivamente). Apesar do número de respondentes ser menor que cinco para estas duas questões específicas (mães ausentes e 
pais namorados), o valor de $p$ para esta associação foi de 0,0059 . Como o cálculo de qui-quadrado considera associação significativa para um "n" maior que cinco, não podemos afirmar que a situação conjugal dos pais, de fato, interfere na iniciação sexual dos adolescentes, embora isso pareça ser sugestivo.

Não houve associação estatisticamente significativa entre renda familiar e iniciação sexual na adolescência, nem entre a escolaridade da mãe e a iniciação sexual na adolescência, e entre a prática de religião do adolescente e sua iniciação sexual.

A tabela 2 revela que a iniciação sexual é menos expressiva entre os adolescentes que nunca trabalharam $(33,1 \%)$, com maior expressão $(55,7 \%)$ entre os que já trabalharam, mas não o fazem atualmente, seguidos pelos que têm vínculo regular e eventual com parceiro. Considerando o valor de $p(0,0160)$ podemos concluir que é provável uma associação positiva entre estas variáveis, ou seja, a iniciação sexual pode ter relação com o fato de $o$ adolescente trabalhar.

O sentimento do adolescente de ser amado pelos pais não apresentou associação significativa com sua iniciação sexual.
Ao analisar com quem o adolescente reside e se o mesmo já teve a primeira relação sexual (Tabela 3), desconsiderando aquele adolescente que já reside com parceiro (pois, consequentemente, já teve sua relação sexual), observa-se que entre os que residem com irmão(ã) (3 adolescentes), dois deles já tiveram sua iniciação sexual $(66,7 \%)$. Entre os que residem somente com o pai (31 adolescentes), 18 deles já tiveram sua primeira relação sexual $(58,1 \%)$. Todavia, esta não é uma associação estatisticamente significante, uma vez que o valor de $p$ foi maior que $0,05(\mathrm{p}=0,1210)$.

Ao associarmos a renda familiar e a frequência com que o adolescente mantém relações sexuais não encontramos associação estatisticamente significativa.

Distribuindo os adolescentes sexualmente ativos ( $\mathrm{n}=198)$ segundo a renda familiar e o fato de já ter tido alguma doença sexualmente transmissível (DST), a proporção dos que já tiveram DST foi maior nas famílias cuja renda era superior a seis salários mínimos $(4,2 \%)$, seguida pelos que não souberam responder sobre a renda $(2,8 \%)$ e pelos adolescentes com renda familiar de quatro e seis salários (2,4\%). Entre os adolescentes que nunca tiveram DST, foram mais frequentes aqueles cujas famílias pos-

Tabela 1 - Distribuição dos adolescentes segundo a situação conjugal dos pais e a iniciação sexual. Cuiabá, 2010.

\begin{tabular}{|c|c|c|c|c|c|c|c|c|}
\hline \multirow{3}{*}{$\begin{array}{l}\text { SITUAÇÃO } \\
\text { CONJUGAL } \\
\text { DOS PAIS }\end{array}$} & \multicolumn{8}{|c|}{ O ADOLESCENTE JÁ TEVE A PRIMEIRA RELAÇÃO SEXUAL } \\
\hline & \multicolumn{2}{|c|}{ Sim } & \multicolumn{2}{|c|}{ Não } & \multicolumn{2}{|c|}{ Branco } & \multicolumn{2}{|c|}{ Total } \\
\hline & $\mathrm{N}$ & $\%$ & $\mathrm{~N}$ & $\%$ & $\mathrm{~N}$ & $\%$ & $\mathrm{~N}$ & $\%$ \\
\hline Casados & 102 & 36,8 & 167 & 60,3 & 8 & 2,9 & 277 & 100 \\
\hline Separados & 74 & 44,8 & 88 & 53,3 & 3 & 1,8 & 165 & 100 \\
\hline Pai viúvo & 2 & 33,3 & 3 & 50,0 & 1 & 16,7 & 6 & 100 \\
\hline Mãe viúva & 4 & 26,7 & 11 & 73,3 & - & - & 15 & 100 \\
\hline Pai ausente & 11 & 47,8 & 12 & 52,2 & - & - & 23 & 100 \\
\hline Mãe ausente & 1 & 100 & - & - & - & - & 1 & 100 \\
\hline Namorados & 4 & 80,0 & - & - & 1 & 20,0 & 5 & 100 \\
\hline Pais falecidos & - & - & 1 & 100 & - & - & 1 & 100 \\
\hline Em branco & - & - & 2 & 66,7 & 1 & 33,3 & 3 & 100 \\
\hline Respostas nulas & - & - & - & - & 3 & 100,0 & 3 & 100 \\
\hline TOTAL & 198 & 39,6 & 284 & 56,9 & 17 & 3,4 & 499 & 100 \\
\hline
\end{tabular}

Tabela 2 - Distribuição dos adolescentes segundo o trabalho e a iniciação sexual. Cuiabá, 2010

\begin{tabular}{|c|c|c|c|c|c|c|c|c|}
\hline \multirow{3}{*}{$\begin{array}{l}\text { SE O ADOLESCENTE } \\
\text { TRABALHA }\end{array}$} & \multicolumn{8}{|c|}{ O ADOLESCENTE JÁ TEVE A PRIMEIRA RELAÇAO SEXUAL } \\
\hline & \multicolumn{2}{|c|}{ Sim } & \multicolumn{2}{|c|}{ Não } & \multicolumn{2}{|c|}{ Em branco } & \multicolumn{2}{|c|}{ Total } \\
\hline & $\mathrm{N}$ & $\%$ & $\mathrm{~N}$ & $\%$ & $\mathrm{~N}$ & $\%$ & $\mathrm{~N}$ & $\%$ \\
\hline Nunca trabalhou & 90 & 33,1 & 176 & 64,7 & 6 & 2,2 & 272 & 100 \\
\hline Atualmente não trabalha & 34 & 55,7 & 25 & 41,0 & 2 & 3,3 & 61 & 100 \\
\hline Trabalha regularmente & 29 & 50,0 & 27 & 46,6 & 2 & 3,4 & 58 & 100 \\
\hline Trabalha eventualmente & 35 & 43,2 & 44 & 54,3 & 2 & 2,5 & 81 & 100 \\
\hline Em branco & 10 & 41,7 & 12 & 50,0 & 2 & 8,3 & 24 & 100 \\
\hline Respostas Nulas & - & - & - & - & 3 & 100 & 3 & 100 \\
\hline TOTAL & 198 & 39,7 & 284 & 56,9 & 17 & 3,4 & 499 & 100 \\
\hline
\end{tabular}


suem renda familiar de 1 a 3 salários mínimos (94,7\%), seguidos pelos que referiram renda familiar de 4 a 6 $(85,7 \%)$ e mais de 6 salários mínimos $(83,3 \%)$, além dos que deixaram a renda em branco $(80,0 \%)$ e os que não souberam referir $(72,2 \%)$. Não responderam se tiveram DST, ou não, $11,1 \%$ dos adolescentes sexualmente ativos. $\mathrm{O}$ valor de $p(0,1113)$ não permite associar a renda familiar com a ocorrência de DST.

Percebe-se, pela tabela 4, que entre os adolescentes em atividade sexual no momento do estudo ( $\mathrm{n}=198)$, a proporção daqueles que utilizava método contraceptivo era mais expressiva entre aqueles cujas famílias possuíam renda familiar de 1 a 3 salários mínimos (80,3\%), enquanto o grupo que não utilizava método contraceptivo era maior nos que possuíam a renda mensal superior a 6 salários mínimos (30,4\%), sem considerar os que não souberam referir a renda familiar. Há que se destacar que o uso de método contraceptivo está referido acima de $60 \%$ em todas as faixas de renda familiar. Considerando o valor de $p(0,0236)$, parece existir uma associação positiva entre renda familiar e uso de método contraceptivo entre os adolescentes pesquisados.
Não foi possível encontrar associação entre renda familiar e a experiência de ter engravidado alguém; entre situação conjugal dos pais/prática de religião e a experiência de ter engravidado alguém entre os meninos; e entre a renda familiar/situação conjugal dos pais/a prática de religião e a experiência de ter ficado grávida, entre as meninas.

De acordo com a tabela 5 , que analisa a presença de violência sexual segunda a renda familiar, observou-se que grande parte dos adolescentes (acima de 70,0\%), independentemente da renda familiar, refere não ter sofrido violência sexual. Entretanto, há que se considerar a presença de violência sexual, mesmo que mínima, na faixa de 1 a $3 \mathrm{SM}$ e de 4 a 6 SM. Entre estes, ainda há relato de ter sofrido o evento não apenas uma vez, mas várias vezes $(1,6 \%$ na faixa de 1 a $3 \mathrm{SM}$ e $2,4 \%$ na faixa de 4 a $6 \mathrm{SM})$. Há, ainda, os adolescentes que não se lembram de ter sofrido violência sexual e os que deixaram esta questão em branco. Segundo o valor de $p(0,0428)$, e como o teste de Qui-quadrado considera para associações um " $n$ " maior que 5, a associação entre renda familiar e violência sexual possui, portanto, valor estatístico significativo.

Tabela 3 - Distribuição dos adolescentes segundo a convivência de outras pessoas na moradia e a iniciação sexual. Cuiabá, 2010

\begin{tabular}{|c|c|c|c|c|c|c|c|c|}
\hline \multirow{3}{*}{$\begin{array}{l}\text { COM QUEM O } \\
\text { ADOLESCENTE } \\
\text { RESIDE }\end{array}$} & \multicolumn{8}{|c|}{ O ADOLESCENTE JÁ TEVE A PRIMEIRA RELAÇÃO SEXUAL } \\
\hline & \multicolumn{2}{|c|}{ Sim } & \multicolumn{2}{|c|}{ Não } & \multicolumn{2}{|c|}{ Branco } & \multicolumn{2}{|c|}{ Total } \\
\hline & $\mathrm{N}$ & $\%$ & $\mathrm{~N}$ & $\%$ & $\mathrm{~N}$ & $\%$ & $\mathrm{~N}$ & $\%$ \\
\hline Pais & 103 & 37,2 & 166 & 59,9 & 8 & 2,9 & 277 & 100 \\
\hline Pai & 18 & 58,1 & 12 & 38,7 & 1 & 3,2 & 31 & 100 \\
\hline Mãe & 62 & 41,1 & 84 & 55,6 & 5 & 3,3 & 151 & 100 \\
\hline $\operatorname{Tia}(\mathrm{o})$ & 2 & 22,2 & 7 & 77,8 & - & - & 9 & 100 \\
\hline Irmão(a) & 2 & 66,7 & 1 & 33,3 & - & - & 3 & 100 \\
\hline Outro parente & 7 & 50,0 & 7 & 50,0 & - & - & 14 & 100 \\
\hline Parceiro & 1 & 100 & - & - & - & - & 1 & 100 \\
\hline Outro & 2 & 40,0 & 3 & 60,0 & - & - & 5 & 100 \\
\hline Em branco & 1 & 20,0 & 4 & 80,0 & - & - & 5 & 100 \\
\hline Respostas Nulas & - & - & - & - & 3 & 100 & 3 & 100 \\
\hline TOTAL & 198 & 39,6 & 284 & 56,9 & 17 & 3,4 & 499 & $\overline{100}$ \\
\hline
\end{tabular}

Tabela 4 - Distribuição dos adolescentes sexualmente ativos segundo a renda familiar e uso de método contraceptivo. Cuiabá, 2010

\begin{tabular}{|c|c|c|c|c|c|c|c|c|}
\hline \multirow{3}{*}{$\begin{array}{l}\text { RENDA } \\
\text { FAMILIAR }\end{array}$} & \multicolumn{8}{|c|}{ USO DE MÉTODO CONTRACEPTIVO } \\
\hline & \multicolumn{2}{|c|}{ Sim } & \multicolumn{2}{|c|}{ Não } & \multicolumn{2}{|c|}{ Branco } & \multicolumn{2}{|c|}{ Total } \\
\hline & $\mathrm{N}$ & $\%$ & $\mathrm{~N}$ & $\%$ & $\mathrm{~N}$ & $\%$ & $\mathrm{~N}$ & $\%$ \\
\hline Menos de $1 \mathrm{SM}$ & 10 & 66,7 & 4 & 26,7 & 1 & 6,7 & 15 & 100 \\
\hline 1 a $3 \mathrm{SM}$ & 61 & 80,3 & 15 & 19,7 & - & - & 76 & 100 \\
\hline 4 a $6 \mathrm{SM}$ & 30 & 71,4 & 8 & 19,0 & 4 & 9,5 & 42 & 100 \\
\hline Mais de $6 \mathrm{SM}$ & 14 & 60,9 & 7 & 30,4 & 2 & 8,7 & 23 & 100 \\
\hline Não sei & 22 & 61,1 & 11 & 30,6 & 3 & 8,3 & 36 & 100 \\
\hline Em branco & 3 & 60,0 & - & - & 2 & 40,0 & 5 & 100 \\
\hline Respostas Nulas & - & - & - & - & 1 & 100 & 1 & 100 \\
\hline TOTAL & 140 & 70,7 & 45 & 22,7 & 13 & 6,6 & 198 & 100 \\
\hline
\end{tabular}

SM - salário mínimo 
Tabela 5 - Distribuição dos adolescentes segundo a renda familiar e se já sofreu alguma violência sexual. Cuiabá, 2010

\begin{tabular}{|c|c|c|c|c|c|c|c|c|c|c|c|c|}
\hline \multirow{3}{*}{$\begin{array}{l}\text { RENDA } \\
\text { FAMILIAR }\end{array}$} & \multicolumn{12}{|c|}{ O ADOLESCENTE JÁ SOFREU VIOLENCIA SEXUAL } \\
\hline & \multirow[b]{2}{*}{$\mathrm{N}$} & \multirow{2}{*}{$\begin{array}{c}1 \text { vez } \\
\%\end{array}$} & \multicolumn{2}{|c|}{ Várias Vezes } & \multicolumn{2}{|c|}{ Não } & \multicolumn{2}{|c|}{ Não lembra } & \multicolumn{2}{|c|}{ Em branco } & \multicolumn{2}{|c|}{ Total } \\
\hline & & & $\mathrm{N}$ & $\%$ & $\mathrm{~N}$ & $\%$ & $\mathrm{~N}$ & $\%$ & $\mathrm{~N}$ & $\%$ & $\mathrm{~N}$ & $\%$ \\
\hline $\begin{array}{l}\text { Menos de } 1 \\
\text { SM }\end{array}$ & - & - & - & - & 37 & 74,0 & - & - & 13 & 26,0 & 50 & 100 \\
\hline 1 a 3 SM & 3 & 1,6 & 1 & 0,5 & 149 & 78,0 & 3 & 1,6 & 35 & 18,3 & 191 & 100 \\
\hline 4 a $6 \mathrm{SM}$ & 4 & 4,8 & 2 & 2,4 & 60 & 71,4 & - & - & 18 & 21,4 & 84 & 100 \\
\hline $\begin{array}{l}\text { Mais de } 6 \\
\text { SM }\end{array}$ & - & - & - & - & 41 & 85,4 & - & - & 7 & 14,6 & 48 & 100 \\
\hline Não sei & - & - & - & - & 75 & 76,5 & 4 & 4,1 & 19 & 19,4 & 98 & 100 \\
\hline Em branco & - & - & - & - & 14 & 56,0 & 1 & 4,0 & 10 & 40,0 & 25 & 100 \\
\hline $\begin{array}{l}\text { Respostas } \\
\text { Nulas } \\
\end{array}$ & - & - & - & - & - & - & - & - & 3 & 100,0 & 3 & 100 \\
\hline TOTAL & 7 & 1,4 & 3 & 0,6 & 376 & 75,3 & 8 & 1,6 & 105 & 21,0 & 499 & 100 \\
\hline
\end{tabular}

\section{DISCUSSÃO}

Quanto à associação entre a situação conjugal dos pais e a iniciação sexual dos adolescentes, autores descrevem a influência da estrutura familiar na sexualidade dos adolescentes como um fator determinante no processo de vivência e experiências adquiridas na fase do desenvolvimento e maturidade sexual ${ }^{(6)}$.

Desta forma, acredita-se que residir com os pais pode ser um fator positivo para adiar a iniciação sexual, uma vez que estes influenciam os filhos e auxiliam no desenvolvimento da maturidade no que se refere ao início da vida sexual ${ }^{(7)}$. Entretanto, é preciso destacar que a simples presença dos pais não é suficiente para adiar a iniciação sexual, sendo necessário haver o diálogo entre pais e filhos e se preservar costumes e valores da família ${ }^{(5)}$.

A respeito da influência da renda familiar sobre a iniciação sexual dos adolescentes, apesar de nosso estudo não ter encontrado associação positiva entre estas variáveis, muitas pesquisas apontam a renda familiar como um dos maiores determinantes para iniciação sexual precoce dos filhos ${ }^{(5)}$. Atualmente, com a ascensão dos jovens à informação, independentemente da classe social, há que se questionar se a renda é fator determinante isolado ou se há outras variáveis envolvidas, tais como as dificuldades e dúvidas dos próprios pais sobre o que e como orientar os filhos sobre sexualidade ${ }^{(8)}$.

Da mesma maneira não houve associação significativa, entre o início da atividade sexual e a escolaridade da mãe. No entanto, autores discutem a influência desta na sexualidade dos adolescentes. Acredita-se que a família constitui-se uma importante fonte de informação para os jovens, tornando-se essencial políticas e investimentos para a melhora da instrução das mães, a fim de melhorar o acesso e a qualidade da informação por elas transmitidas ${ }^{(5)}$.
Em relação ao trabalho na adolescência, um estudo sobre fatores associados à iniciação sexual de adolescentes constatou que aqueles que já trabalham têm uma iniciação da vida sexual mais precoce em relação aos que não trabalham ${ }^{(9)}$. O que corrobora nossos achados, em que a proporção de adolescentes sexualmente ativos foi maior entre os que já trabalharam ou o faziam regularmente e até mesmo eventualmente. Neste sentido, há que se ressaltar a importância de iniciativas públicas que mantenham o jovem na escola, sem necessidade do mesmo trabalhar, uma vez que se investindo na educação do jovem, este poderá ter uma melhor formação e informação sobre a sexualidade ${ }^{(10)}$.

Entre os adolescentes pesquisados não houve associação significativa entre a iniciação sexual e os sentimentos de serem amados pelos pais. No entanto, autores $^{(7)}$ mencionam que, ao se sentirem amados por seus pais, os adolescentes sentem-se protegidos e compreendidos, existindo então uma melhor comunicação e diálogo entre pais e filhos, o que pode contribuir para uma iniciação sexual mais tardia. Acredita- se que o fato de não se sentirem amados faz com que os adolescentes busquem na atividade sexual uma forma de compensar essa carência afetiva. Quando existe o afeto, a confiança e admiração dos pais e valores morais da família, esses influem sobre os desejos do adolescente, ocasionando um adiamento da sua iniciação sexual ${ }^{(5)}$.

Quanto ao fato dos adolescentes ( $\mathrm{n}=198)$, que já tiveram relação sexual, morarem apenas com o pai (18 adolescentes) ou só com a mãe (62 adolescentes), um estudo afirma que muitos dos jovens pertencentes a famílias desfeitas se tornam mais vulneráveis àqueles que possam lhes dar algum tipo de afeto, estimulando o adolescente numa situação de busca pelo outro como forma de preencher suas carências afetivas de pai e mãe ${ }^{(11)}$. 
Apesar do presente estudo não ter encontrado associação entre renda familiar e a frequência de relações sexuais dos adolescentes, a literatura aponta que aqueles que possuem maior renda se relacionam sexualmente com maior frequência, pois jovens com melhores condições financeiras sentem-se mais autoconfiantes com relação à sexualidade por terem melhor orientação e acesso sobre o assunto, enquanto que aqueles que possuem a renda mais baixa precisam melhorar a renda da família através do trabalho, o que de alguma forma ocupa maior parte do seu tempo, fazendo com que pratiquem sexo com menor frequência ${ }^{(9)}$.

Quanto às DST entre adolescentes, estudiosos ${ }^{(12)}$ afirmam que, normalmente, a incidência está relacionada a baixos níveis socioeconômicos, o que diverge dos nossos achados, nos quais, de 192 adolescentes, a DST ocorreu em $7 \%$ daqueles com renda familiar entre 4 e mais de $6 \mathrm{SM}$. Porém, há que se considerar que não apenas a renda está associada ao agravo, mas, principalmente, com a autoconfiança entre os adolescentes, característica desta fase, o que faz com que se sintam invulneráveis, expondo-se a riscos sem prever suas consequências.

Sobre a renda familiar e o uso de método contraceptivo, muitos estudos demonstram que a probabilidade dos adolescentes iniciar a vida sexual sem a utilização dos métodos contraceptivos, com uma possível gravidez precoce, é maior entre aqueles cuja renda familiar é baixa ${ }^{(7,9)}$. Porém, a nossa casuística não foi concordante com a literatura, o que suscita a reflexão de que possa haver outros fatores envolvidos na contracepção e gestação precoce que não apenas a renda familiar ou a pobreza. Neste sentido, um estudo mostra que a gravidez na adolescência também se relaciona com as expectativas familiares e sociais interrompidas, com o amor e o desamor nas relações interpessoais ${ }^{(13)}$, o que suscita a necessidade de resgatar as relações familiares. Apesar de não termos encontrado associação positiva entre renda familiar e situação conjugal dos pais com a experiência de gravidez, tanto a experiência do menino ao engravidar sua companheira quanto da menina ao ficar grávida, estudos apontam que aqueles adolescentes que possuem a convivência com os pais casados, ou com pelo menos um deles, tendem a ter a experiência de gravidez mais tardiamente ${ }^{(14-15)}$.

Os resultados da pesquisa não mostram significância na associação entre religião, situação conjugal dos pais e gravidez, mas um estudo verificou que tanto os jovens do sexo masculino quanto feminino, e que eram adeptos de alguma religião, tinham a experiência sexual bem mais tardia e, consequentemente, a postergação da experiência de gravidez ${ }^{(10)}$. Pesquisadores afirmam que a religiosidade e a espiritualidade exercem uma influência positiva sobre as condutas de saúde na adolescência, podendo assim ser um importante mediador de adiamento da exposição desses adolescentes a fatores de risco ${ }^{(16)}$. Embora a vivência da sexualidade seja de caráter íntimo, muitos jovens, ao se tornar adeptos de alguma religião passam a se orientar por regras e valores estabelecidos pelo grupo religioso no qual estão inseridos ${ }^{(17)}$.

Quando à violência sexual, destaca-se no presente estudo o fato de uma grande parte dos adolescentes deixar esta resposta em branco, ou referir não se lembrar se sofreu violência sexual, o que pode estar associado ao caráter silencioso e insidioso da violência sexual, o que faz com que ainda seja pouco assumida e denunciada pelas suas vítimas ${ }^{(18)}$. Destaca-se que a simples ocorrência da violência sexual entre os adolescentes estudados corrobora o fato de que a violência constituise em fenômeno de preocupação mundial, uma vez que atinge todas as classes sociais, de ambos os sexos e diferentes faixas etárias, com repercussões significativas tanto para as vítimas como para a sociedade e serviços de saúde ${ }^{(19)}$.

Em estudos realizados com adolescentes vítimas de violência sexual, a maior proporção se verificou entre aqueles que possuem renda familiar inferior a dois salários mínimos, associando assim pobreza com violência ${ }^{(20)}, \mathrm{o}$ que não se observou na presente investigação. Isto nos leva à reflexão de que há outros fatores associados com o fenômeno, relativizando-se a ideia de que a violência acomete, em maior quantidade, aqueles adolescentes que pertencem à classes sociais mais baixas.

\section{CONCLUSÃo}

O presente estudo teve como objetivo estudar as questões socioeconômico-familiares relativas à atividade sexual de adolescentes. Com base nos resultados percebe-se a necessidade de compreender melhor os fatores envolvidos no exercício da sexualidade, uma vez que alguns dos resultados são divergentes da literatura, sugerindo que a renda familiar não seja o único fator vinculado à presença de doenças sexualmente transmissíveis, gravidez precoce e violência sexual. Talvez, as divergências encontradas possam ser atribuídas à realidade local, uma vez que, conforme o desenvolvimento de cada região, os dados apresentam-se diferenciados. Desta forma, sugere-se a realização de novas abordagens de pesquisa nesta mesma população de estudo, a fim de compreender melhor os fatores que contribuíram para os achados divergentes da literatura. 
Assim, ressalta-se a importância do envolvimento dos profissionais dos diferentes setores, como da saúde, educação, direito, serviço social e áreas afins, para que possibilitem e desenvolvam projetos que atendam às necessidades dos adolescentes quanto à orientação sexual, revelada na presente investigação como necessária.

Destaca-se, ainda, a importância de se realizar uma assistência integral à adolescência por equipe multiprofissional, visando construir um vínculo entre Sistema de Saúde, adolescentes e suas famílias. A realização de práticas educativas, principalmente nas escolas e unidades de saúde, pode contribuir significativamente, uma vez que os jovens estão se iniciando cada vez mais cedo na prática sexual, independentemente da renda familiar, tornando-se mais vulneráveis às DST e à gravidez precoce. Neste sentido, o profissional, tanto de saúde como de educação, revela-se como agente transformador na efetividade da assistência integral à saúde sexual e reprodutiva do adolescente.

Espera-se que esse estudo contribua com os gestores, educadores e profissionais, para que possam trabalhar com os adolescentes nessa fase de muitas transformações e dúvidas. Sugere-se, ainda, a realização de pesquisas sobre as questões socioeconômico-familiares associadas à prática sexual dos adolescentes, uma vez que o tema é pouco discutido na literatura científica.

\section{REFERÊNCIAS}

1. Camargo EAI, Ferrari RAP. Adolescentes: conhecimentos sobre sexualidade antes e após a participação em oficinas de prevenção. Ciênc Saúde Coletiva. 2009; 14(3):937-45.

2. Albino GC, Vitalle MSS, Schussel EY, Batista NA. A sexualidade pelo olhar das jovens: contribuições para a prática do médico de adolescentes. Rev Paul Pediatr. 2005;23(3):124-9.

3. Falcão DVS, Salomão NMR. Mães adolescentes de baixa renda: um estudo sobre as relações familiares. Arq Bras Psicol. 2006;58(2):11-21.

4. Gaspar T, Matos MG, Ribeiro JL, Leal I. Qualidade de vida em crianças e adolescente: impacto da migração e estatuto socioeconômico. Braz J Health. 2010;1(1):136-52.

5. Souza LB, Fernandes JFP, Barroso MGT. Sexualidade na adolescência: análise da influência de fatores culturais presentes no contexto familiar. Acta Paul Enferm. 2006;19(4):408-13.
6. Fonseca AD, Gomes VLO, Teixeira KC. Percepção de adolescentes sobre uma ação educativa em orientação sexual realizada por acadêmicos(as) de enfermagem. Esc Anna Nery. 2010;14(2):330-7.

7. Borges ALV, Latorre MRDO, Schor N. Fatores associados ao início da vida sexual de adolescentes matriculados em uma unidade de saúde da família da zona leste do município de São Paulo, Brasil. Cad Saúde Pública. 2007;23(7):1583-94.

8. Romero KT, Medeiros EHGR, Vitalle MSS, Wehba J. O conhecimento das adolescentes sobre questões relacionadas ao sexo. Rev Ass Med Bras. 2007;53(1):14-9.

9. Bergamim MD, Borges ALV. Fatores associados à iniciação sexual entre adolescentes da zona oeste do município de São Paulo. Rev Gaúcha Enferm. 2009;30(3):420-8.

10. Cruzeiro ALS, Souza LDM, Silva RA, Horta BL, Muenzer RM, Faria AD, et al. Iniciação sexual entre adolescentes de pelotas, Rio Grande do Sul. Rev Bras Crescimento Desenvolv Hum. 2008;18(2):116-23.

11. Oriá MOB, Alves MDS. Adolescente com papiloma vírus humano no contexto familiar. Rev Enferm UERJ. 2004;12(1):44-8.

12. Custódio G, Massuti AM, Schuelter-Trevisol F, Trevisol DJ. Comportamento sexual e de risco para DST e gravidez em adolescentes. DST - J Bras Doenças Sex Transm. 2009;1(1):60-2.

13. Machado M, Zagonel I. O processo de cuidar da adolescente que vivencia a transição ao papel materno. Cogitare Enferm. 2005;8(2):26-33.

14. Dias AB, Aquino EML. Maternidade e paternidade na adolescência: algumas constatações em três cidades do Brasil. Cad Saúde Pública. 2006;22(7):1447-58.

15. Ximenes Neto FRG, Dias MSA, Rocha J, Cunha ICKO. Gravidez na adolescência: motivos e percepções de adolescentes. Rev Bras Enferm. 2007;60(3):279-85.

16. Bezerra J, Barros MVG, Tenório MCM, Tassitano RM, Barros SSH, Hallal PC. Religiosidade, consumo de bebidas alcoólicas e tabagismo em adolescentes. Rev Panam Salud Publica. 2009;26(5):440-6.

17. Paiva V, Aranha F, Bastos F. Opiniões e atitudes em relação à sexualidade: pesquisa de âmbito nacional, Brasil 2005. Rev Saúde Pública. 2008;42(1):54-64. 
18. Ribeiro MA, Ferriani MGC, Reis JN. Violência sexual contra crianças e adolescentes: características relativas à vitimização nas relações familiares. Cad Saude Publica. 2004;20(2):456-64.

19. Brito AM, Zanetta DM, Mendonça RC, Barison SZ, Andrade VAG. Violência doméstica contra crianças e adolescentes: estudo de um programa de intervenção. Ciênc Saúde Colet. 2005;10(1):143-9.

20. Gomes MLM, Falbo Neto GH, Viana CH, Silva MA. Perfil clínico-epidemiológico de crianças e adolescentes do sexo feminino vítimas de violência atendidas em um serviço de apoio à mulher, Recife, Pernambuco. Rev Bras Saúde Matern Infant. 2006;6(1):527-34. 